\title{
Prevalence, Antibiotic Susceptibility Profile, and Associated Risk Factors of Salmonella Isolate among Diarrheal Patients Visiting Dessie Referral Hospital, Northeast Ethiopia
}

\author{
Samuel Chane Teferi (iD \\ College of Natural and Computational Sciences, Department of Biology, Salale University, Fitche, Ethiopia \\ Correspondence should be addressed to Samuel Chane Teferi; samuelchane19@gmail.com
}

Received 15 August 2020; Revised 10 September 2020; Accepted 7 October 2020; Published 22 October 2020

Academic Editor: Luigi Santacroce

Copyright (c) 2020 Samuel Chane Teferi. This is an open access article distributed under the Creative Commons Attribution License, which permits unrestricted use, distribution, and reproduction in any medium, provided the original work is properly cited.

\begin{abstract}
Salmonellosis remains an important public health problem worldwide, particularly in the developing countries such as Ethiopia. A cross-sectional study was conducted to determine the prevalence, antibiotic susceptibility profile, and associated risk factors of Salmonella isolate among diarrheal patients who were visiting Dessie Referral Hospital, Dessie, Northeast Ethiopia, from November 2016 to January 2017. 384 stool samples were collected using sterile stool cups. Out of these, 20 (5.21\%) were found to be positive for Salmonella species. The distribution of positive samples among the three age groups indicated that Salmonella species were predominantly prevalent in the age group of three months ( 0.25 years) to 4 years old patients. Abdominal pain, vomiting, watery consistency of stool, and 1-5 days of diarrhea were the clinical features that were significantly associated with salmonellosis. Eating raw vegetables and fruits, consumption of street-vended foods, cohabitation of animals, using water from the unprotected source, absence of latrine, and consumption of raw products of animals such as eggs and raw milk were the risk factors that were significantly associated with the prevalence of Salmonella species. The antibiotic sensitivity test was performed for the isolated Salmonella species against 5 currently recommended antibiotics. The antimicrobial sensitivity study carried out using the Kirby-Bauer disk diffusion method showed that $100 \%$ of Salmonella isolates were sensitive to ciprofloxacin and $80 \%$ sensitive to nalidixic acid, respectively. Among them, twenty isolated Salmonella species resistant to ampicillin, tetracycline, and trimethoprim-sulfamethoxazole were $100 \%, 85 \%$, and $80 \%$, respectively. This study revealed that Salmonella species were prevalent among diarrheal patients who were visiting Dessie Referral Hospital, and therefore, routine diagnosis of patients with diarrhea cases is required, and drugs must be prescribed after performing the sensitivity test, or checking the updated information on the local antibiotics pattern is always necessary.
\end{abstract}

\section{Introduction}

Diarrhea is a condition of having three or more loose or liquid bowel movement per day [1]. Human diarrheal diseases have been recognized since the beginning of civilization and remain one of the most prevalent public health problems of today. Diarrheal infection spreads through the ingestion of contaminated food or drinking water or person to person contact as a result of poor hygiene. According to [1], diarrheal disease is the second leading cause of death in children under five years old. Each year diarrhea kills around 525,000 under-five children. $12 \%$ from the estimated 3.6 million child deaths in 2010 were attributed to diarrheal diseases. The incidence of childhood diarrhea in Africa has also decreased from 4.2 to 3.3 episodes per child-year from 1990 to 2010, but sub-Saharan Africa still accounts for onethird of diarrheal episodes yearly (500 million of 1.7 billion worldwide), with the highest incidence among children of 6-11 months of age [2]. In Ethiopia, as in other sub-Saharan African countries, morbidity from diarrheal diseases is a serious health problem, and according to [3], Salmonella was one of the major causes of diarrhea in humans. Diarrhea in children is the second leading top ten causes of death that accounted 8\% in Ethiopia [4]. 
Diarrhea can be caused by a wide variety of bacterial, viral, and protozoan pathogens. The most important of these are Salmonella spp, Shigella spp, Vibrio cholera, Entamoeba histolytica, Norovirus, Giardia lamblia, Escherichia coli, Campylobacter jejuni, Cryptosporidium sp., and Rotavirus [5].

Salmonellosis, the disease caused by Salmonella, is one of the most frequently occurring foodborne diseases worldwide [6]. As a result, it continues to be a major health burden worldwide. In Ethiopia, 10.5\% Salmonella prevalence was reported in Butajira [7], 6.2\% Salmonella prevalence was reported in Jimma Health Center [8], a prevalence of $14.6 \%$ Salmonella infection was reported in Harar among patients who were admitted to hospital [9], 3.95\% Salmonella infection was reported in children of Addis Ababa [10], and 2.5\% was reported in Hawassa [11].

The majority of Salmonella cases occur as the result of ingesting contaminated food or water, but it can also be transmitted from person to person via the oral-fecal route. Salmonella can also be acquired by contact with domestic animals and their food products, farm animals or animals in petting zoo, and exotic pets such as turtles, hedgehogs, and iguanas [12].

A study in Ethiopia indicated that there is an increase in antimicrobial resistant in pathogenic bacteria causing diarrheal disease because of easy affordability of antibiotics in pharmacies and misuse [3, 13]. Therefore, antimicrobial resistance in bacterial pathogens causing diarrheal diseases makes it difficult to treat patients. In Dessie town, particularly the rural area of Dessie, there are a number of risk factors, which lead to gastrointestinal infections. For example, poor sanitary conditions, cattle living together with people, drinking raw milk and eating raw meat, and consumption of street-vended foods are common practices. These factors are directly or indirectly related to consumption of water and foods. According to [12], contaminated water and foods are responsible for initiating salmonellosis. So, the objective of this study was to investigate the prevalence, antibiotic susceptibility profile, and associated risk factors of Salmonella among diarrheal patients visiting Dessie Referral Hospital, Dessie town, Northeast Ethiopia.

\section{Materials and Methods}

2.1. Study Design. A cross-sectional study was carried out from November 2016 to January 2017 on diarrheal patients visiting Dessie Referral Hospital, Dessie. A pretested structured questionnaire was administered to patients or to their parents or guardians to collect information on sociodemographic and risk factors including sex, age, educational status, place of residence, source of drinking water, latrine usage, personal hygiene practice, and clinical features of the study subjects.

2.2. Study Population. The study included all diarrheal patients visiting the outpatient department of Dessie Referral Hospital. The sample size $(n)$ was determined through a single proportion formula by taking an estimated prevalence of 0.5 , since there is no previous investigation on the same title in the study area and margin of error 0.05. Sample size was determined using the following formula [14].

$$
n=\frac{(Z \alpha / 2)^{2} P(1-P)}{d^{2}}
$$

where $n$ is the number of sample size. $Z \alpha / 2$ is the standard score corresponding to $95 \%$ confidence level, i.e., 1.96. $P$ is the prevalence of Salmonella. $D$ is the marginal of error between the sample and the population. So, the calculated sample size for this study was 384 .

2.3. Sampling Method. The sources of population were all diarrheal patients attending Dessie Referral Hospital. Those patients who had diarrhea and included in the sample were coded and assisted supervised by physicians or nurses in the outpatient department to fill the structured questionnaires. Then, fresh stool specimens were collected from diarrheal cases at Dessie Referral Hospital and transported using sterile stool cups to Dessie Regional Laboratory for analysis.

2.4. Isolation and Identification of Salmonella Species. About $1 \mathrm{~g}$ of stool sample was added into a tube containing selenite $F$ broth. Selenite broth was incubated aerobically at $37^{\circ} \mathrm{C}$ for 18 hours [15]. For ideal isolation of Salmonella, two different selective media, general purpose medium with low sensitivity MacConkey agar and a high selective xylose-lysine desoxycholate agar (Oxoid, England), were used. A loopful of faecal suspension from enrichment media or selenite broth was subcultured on xylose-lysine desoxycholate agar and MacConkey agar. Then, plates were incubated aerobically at $35-37^{\circ} \mathrm{C}$ for $18-24$ hours. Typical colorless colonies on MacConkey agar and colonies with a black center and a lightly transparent zone of reddish color due to the color change of the indicator were picked, and a series of biochemical tests were performed for further identification [15]. Those are the urease test (HiMedia, India), triple sugar iron agar (TSI) test (HiMedia, India), lysine iron agar (LIA) test (Oxoid, England), motility test (Oxoid, England), indole test (Oxoid, England), and citrate utilization test (Oxoid, England).

2.5. Antimicrobial Susceptibility Test. The antibiotic susceptibility profile of Salmonella isolates was determined for the commonly used antibiotics (Oxoid Ltd., Basingstoke, England). Each isolate was tested for the selected antimicrobial agents with their respective concentrations such as ampicillin $(10 \mu \mathrm{g})$, tetracycline $(30 \mu \mathrm{g})$, trimethoprim-sulfamethoxazole $(1.25 / 23.75 \mu \mathrm{g})$, nalidixic acid $(30 \mu \mathrm{g})$, and ciprofloxacin $(100 \mu \mathrm{g})$. Using a sterile wire loop, 3-5 well isolated colonies were picked and emulsified in $5 \mathrm{ml}$ nutrient broth. The prepared turbidity was matched with a turbidity standard (0.5 McFarland) to have an equivalent suspension. A sterile swab was used to inoculate the suspension by streaking on the prepared and dried Mueller-Hinton agar plate evenly. It was then allowed to stay for 3-5 minutes. 
Sterile forceps were used to place the antimicrobial discs on the inoculated plates [16].

Within 30 minutes after applying the disc, the plates were incubated at $35^{\circ} \mathrm{C}$ for $16-18$ hours. After incubation, diameters of the zone of inhibition was measured to the nearest millimeter using a metallic caliper, and the isolates were classified as sensitive, intermediate, and resistant according to the standardized table provided by [16]. The standard reference strain, Escherichia coli (American type culture collection 25922), was used as a control for the study.

2.6. Data Analysis. The data were entered and analyzed using the Statistical Package for Social Sciences (SPSS) version 16 software. The prevalence of Salmonella species was calculated as percentage, and results are presented in tables. Statistical significance of the association was measured by using the chi-square test (association between selected risk factors and Salmonella infection). $P$ values less than 0.05 were considered as statistically significant.

2.7. Ethical Considerations. Institutional consent was acquired through communications made with Dessie Referral Hospital before conducting the study. The medical director of Dessie Referral Hospital was briefed first about the study before meeting with patients. Both oral and written informed consents were obtained from the parents or guardians of the study subjects before administration of the study. The participation of patients in this study was purely a voluntary activity, and their right not to participate was respected. Issues of confidentiality and anonymity were also maintained.

\section{Results and Discussion}

Three hundred eighty-four $(n=384)$ diarrheal patients attending the outpatient department of Dessie Referral Hospital were examined for Salmonella spp during the threemonth study period. Distribution of the study participants by sex and age is shown in Table 1. Age group category was based on the Dessie Regional Health Bureau data record system on diarrheal diseases prevalence, where $0-4,4-14$, and $\geq 15$ age groups are reported as under-five children, young children, and adults, respectively.

Highest frequency of diarrheal diseases occurred in the age group of 3 months to four years (Table 1). This was consistent with [2] who reported a highest incidence among children of 6-11 months of age.

3.1. Prevalence of Salmonella Species. The prevalence of Salmonella isolates in stool samples of this study, 5.21\% (20/ 384 ), is comparable to the findings of previous studies made in China by [17] who reported a prevalence of $5.8 \%$ and $4.8 \%$ Salmonella in the years 2006 and 2007, respectively, and $5.3 \%$ a study performed at Jimma and Addis Ababa, Ethiopia [13].

On the other hand, a lower prevalence was reported by [18] from Djibouti who reported a prevalence rate of $2.9 \%$ and 3.3\% from Lagos, Nigeria [19], 3\% Botswana [20], and $1.08 \%$ [21], 2.5\% [11], and 3.95\% [10] from Ethiopia. However, the isolation rate in this study was found to be lower than the $6.2 \%$ Salmonella prevalence reported by [8], a study conducted on the prevalence of intestinal parasite, Shigella and Salmonella species among diarrheal children in Jimma health center, Jimma, southwest Ethiopia, and much lower than the $10.5 \%$ and $14.6 \%, 16.7 \%$, and $17 \%$ prevalence of Salmonella reported by [7, 9, 22, 23] in Ethiopia, Mexico, and Nigeria, respectively.

The prevalence of Salmonella infection by age and sex in the present study is also shown in Table 1 . As can be seen from the table, Salmonella infection was seen in both male and female patients, but it was statically insignificant with 0.800 of the chi-square value and $P$ value of 0.371 . This implies that both sexes are equally at risk of Salmonella infection. Among the 20 patients infected with Salmonella species, $12(60 \%)$ were females and $8(40 \%)$ were males, which gave an overall male to female ratio of 1:1.5.

As indicated in Table 1, Salmonella were isolated in all age groups, and a statistically significant difference existed between the prevalence of the different age groups $(P=0.022)$. Out of 20 positive Salmonella isolates, more Salmonella infection (12/20 or $60 \%)$ was observed in children of $0-4$ years of age than in those young children aged between 5 and 14 years (30\%) and adults above 15 years (10\%).

\subsection{Association of Salmonella Infection and Identified Risk} Factors. The association of Salmonella infection and prevalence of Salmonella spp. are shown in Table 2. The data generally show that infection with Salmonella spp. is associated significantly with the absence of latrine $\left(X^{2}=7.200\right.$, $P=0.007)$, drinking unprotected water $\left(X^{2}=5.000\right.$, $P=0.025)$, consumption of egg $\left(X^{2}=5.000, P=0.025\right)$, cohabitation with domestic animals $\left(X^{2}=7.200, P=0.007\right)$, consumption of raw milk $\left(X^{2}=9.800, P=0.002\right)$, consumption of raw vegetables and fruits $\left(X^{2}=16.200\right.$, $P=0.000)$, and consumption of street-vended foods $\left(X^{2}=12.800, P=0.000\right)$.

In this study, there was a significant link between the absence of latrine and infection with Salmonella. Contracting salmonellosis and poor living and housing conditions was significantly associated. This finding was typical to the situation, throughout the developing world [24].

Association of drinking from the unprotected water source and prevalence of salmonellosis $\left(X^{2}=5.000\right.$ and $P=0.025)$ was significant. From 20/384 positive cases for salmonellosis, those who used protected water had less risk of contracting salmonellosis than those who used from the unprotected source (Table 2). This can be due to the high level of contamination of water by Salmonella from the environment. The finding was in line with [25] who reported that the transmission of Salmonella typhi in humans was associated with the ingestion of contaminated water. Moreover, a study has shown that exposure to contaminated water is known to be associated with diarrhea caused by ingestion of microorganisms [26]. 
Table 1: Prevalence of Salmonella spp. and sociodemographic characteristics of the study participants $(n=384)$.

\begin{tabular}{|c|c|c|c|c|}
\hline \multicolumn{2}{|c|}{ Sociodemographic characteristics } & Total numbers examined & No. of positives (\%) & $P$ values \\
\hline \multirow{3}{*}{ Age (years) } & $0.25-4$ & 180 & $12(60)$ & \multirow{3}{*}{$0.022^{* * *}$} \\
\hline & $5-14$ & 120 & $6(30)$ & \\
\hline & $>15$ & 84 & $2(10)$ & \\
\hline \multirow{2}{*}{ Gender } & Male & 181 & $8(40)$ & \multirow{2}{*}{0.371} \\
\hline & Female & 203 & $12(60)$ & \\
\hline \multirow{2}{*}{ Place of residence } & Urban & 171 & 7 & \multirow{2}{*}{0.180} \\
\hline & Rural & 213 & 13 & \\
\hline \multirow{2}{*}{ Educational status } & Literate & 142 & 12 & \multirow{2}{*}{0.371} \\
\hline & Illiterate & 242 & 8 & \\
\hline
\end{tabular}

TABle 2: Prevalence of Salmonella spp. and associated risk factors in the study area in 2016/17.

\begin{tabular}{|c|c|c|c|c|c|}
\hline \multirow{2}{*}{ Factors } & \multicolumn{2}{|c|}{ Salmonella species } & \multirow{2}{*}{$X^{2}$} & \multirow{2}{*}{ Df } & \multirow{2}{*}{$P$ value } \\
\hline & Positive (\%) & Negative (\%) & & & \\
\hline \multicolumn{6}{|l|}{ Residence } \\
\hline Urban & $7(4.1)$ & $164(95.9)$ & \multirow{2}{*}{1.800} & \multirow{2}{*}{1} & \multirow{2}{*}{0.180} \\
\hline Rural & $13(6.1)$ & $200(93.9)$ & & & \\
\hline \multicolumn{6}{|c|}{ Educational status } \\
\hline Literate & $12(8.5)$ & $130(91.5)$ & \multirow{2}{*}{0.800} & \multirow{2}{*}{1} & \multirow{2}{*}{0.371} \\
\hline Illiterate & $8(3.3)$ & $234(96.7)$ & & & \\
\hline \multicolumn{6}{|l|}{ Latrine } \\
\hline Present & $3(1.4)$ & $210(98.6)$ & \multirow{2}{*}{7.200} & \multirow{2}{*}{1} & \multirow{2}{*}{$0.007^{* * *}$} \\
\hline Absent & $17(10)$ & $154(90)$ & & & \\
\hline \multicolumn{6}{|l|}{ Drinking water } \\
\hline Protected & $5(2.5)$ & $194(97.5)$ & \multirow{2}{*}{5.000} & \multirow{2}{*}{1} & \multirow{2}{*}{$0.025^{* * *}$} \\
\hline Unprotected & $15(8.1)$ & $170(91.9)$ & & & \\
\hline \multicolumn{6}{|c|}{ Domestic animals } \\
\hline Present & $14(6.1)$ & $214(93.9)$ & \multirow{2}{*}{3.200} & \multirow{2}{*}{1} & \multirow{2}{*}{0.074} \\
\hline Absent & $6(3.8)$ & $150(96.2)$ & & & \\
\hline \multicolumn{6}{|c|}{ Domestic animal house } \\
\hline Separate & $4(2.2)$ & $174(97.8)$ & \multirow{2}{*}{7.200} & \multirow{2}{*}{1} & \multirow{2}{*}{$0.007^{* * *}$} \\
\hline Cohabit & $16(7.8)$ & $190(92.2)$ & & & \\
\hline Raw milk & & & & & \\
\hline Used & $17(14.5)$ & $100(85.5)$ & & & \\
\hline Unused & $3(1.1)$ & $264(98.9)$ & 9.800 & 1 & $0.002^{* x^{*}}$ \\
\hline Raw meat & & & & & \\
\hline Used & $13(6.1)$ & $200(93.9)$ & & & \\
\hline Unused & $7(4.1)$ & $164(95.9)$ & 1.800 & 1 & 0.180 \\
\hline Raw vegetables & & & & & \\
\hline Used & $19(5.9)$ & $302(94.1)$ & & & \\
\hline Unused & $1(1.6)$ & $62(98.4)$ & 16.200 & 1 & $0.000^{* 2 * 3}$ \\
\hline Consumption o & foods & & & & \\
\hline Used & $18(8.5)$ & $193(91.5)$ & 12800 & 1 & $0000 * * *$ \\
\hline Unused & $2(1.2)$ & $171(98.8)$ & 12.800 & 1 & 0.000 \\
\hline Raw egg & & & & & \\
\hline Used & $15(6)$ & $234(94)$ & 5000 & 1 & $0025 * * *$ \\
\hline Unused & $5(3.7)$ & $130(96.3)$ & 5.000 & 1 & 0.025 \\
\hline
\end{tabular}

*** Significant at $P<0.05$; Df, degree of freedom; $X^{2}$, chi-square.

Salmonella spp. infection was shown to be significantly associated with those who had domestic animals (cohabitation); the present finding was in line with [9] from Harar, Ethiopia. This finding suggests the fact that Salmonella spp. are important human and animal pathogens and acquired from contact with domestic animal and their food products [12]. Besides, the aquatic environment is liable to contamination through colistin-treated animal faeces via the use of manures in feeding farmed fish [27].

Raw milk and raw milk products consumption from a dairy may result in infection by Salmonella and many other pathogenic bacteria [28]. The present finding also revealed a significant association between raw milk consumption and contracting of Salmonella infection. This is comparable to 
the findings in Pennsylvania who reported the transmission of enteric pathogen infections to humans via the consumption of raw milk [29].

In the present study, an association between salmonellosis and consumption of raw vegetables and fruits from unhygienic sources were significant (Table 2). In recent years, fresh produce such as fruits and vegetables have gained concern as vehicles of transmission where contamination can occur at multiple steps along the food chain [30].

The prevalence of Salmonella spp. was also associated with the consumption of street-vended foods, which was consistent with a report from Ghana by $[9,31]$ from Harar, Ethiopia. This might be due to street-vended foods sold under unhygienic conditions in the study area. Salmonella has been known as the most important foodborne pathogen, which can infect humans via consuming contaminated food [32]. Moreover, identifying genetic subpopulations and understanding their epidemiology may contribute to efforts invested in the prevention and mitigation of these important foodborne pathogens [33].

According to [34], consumption of contaminated products such as meat and eggs initiate salmonellosis in human. In the present study, a significant association $\left(X^{2}=5.000, P=0.025\right)$ was found between consumption of raw eggs and Salmonella infection. Those patients who consumed raw egg were likely contracting salmonellosis than those who did not consume such products. Moreover, according to [35], human and poultry isolates bore more antimicrobial resistance and virulence genes, and the meat pathway may be an important source of human infection by some clades of Salmonella enteritidis ST11 in East Africa, and according to [36], the emergence and spread of new strains of zoonotic bacteria, such as multidrug resistant (MDR) Salmonella infantis, represent a growing health risk for humans in and outside Europe due to foodborne infections of poultry meat origin.

There was no statistically significant association between residence, educational status, presence of domestic animal, and raw meat on one hand and infection with Salmonella on the other hand as indicated in Table 2.

3.3. Association between the Prevalence of Salmonella spp. and Clinical Features. Association between the prevalence of Salmonella spp. and clinical features are summarized in Table 3. In this study, out of twenty Salmonella-positive patients, the majority had abdominal pain followed by vomiting and fever, which accounted for $90 \%, 80 \%$, and $70 \%$ of the patients, respectively. This result was comparable with the study conducted in Harar by [9] who reported $85.7 \%$ abdominal pain and [11] showed 50\% abdominal pain. There was a significant association between abdominal pain, vomiting, and Salmonella infection $\left(X^{2}=12.800,7.200\right.$ and $P=0.000,0.007$, respectively), whereas there was no significant association in fever (Table 3 ).

The present study showed that there was a significant association between consistency of stools, duration of diarrhea, and positivity of Salmonella infection $\left(X^{2}=13.600\right.$, 7.600 and $P=0.004,0.022$, respectively) as the majority $60 \%$
(12) of the stool samples were watery diarrhea in contrast to [37] in Harar who reported that no Salmonella was isolated from watery diarrhea. But the present study is comparable with [9], a study performed in Harar who reported 28.6\% watery diarrhea, and $82.4 \%$ Salmonella was isolated from watery diarrhea in a study performed in Addis Ababa [38].

The remaining $20 \%(4), 10 \%(2)$, and $10 \%$ (2) of the isolated stool samples were mucoid, bloody, and mixed (blood and mucus), respectively (Table 3 ). This may reflect the underlying variation in signs and symptoms with strain differences from place to place.

Out of the twenty isolates, $12(60 \%)$ were having 1-5 days duration of diarrhea, which is consistent with [11] who reported $75 \%$ and [9] who reported $71.4 \%$ of the isolate was from 1 to 5 days. Whereas the remaining 30\% and $10 \%$ of Salmonella isolate were 6-10 and 11-15 days of diarrhea, respectively. The use of clinical signs and symptoms is therefore very important in helping to identify patients with salmonellosis.

3.4. Antimicrobial Susceptibility Test. The antimicrobial susceptibility tests were performed on all Salmonella isolates using the disk diffusion method, and the results are presented in Table 4. Among 20 Salmonella isolates, 20 (100\%), $17(85 \%), 16(80 \%)$, and $3(15 \%)$ were resistance to ampicillin, tetracycline, trimethoprim-sulphamethoxazole, and nalidixic acid, respectively. But all Salmonella isolates showed $100 \%$ susceptibility to ciprofloxacin.

$3(15 \%), 2(10 \%)$, and $1(5 \%)$ of the isolate showed an intermediate resistance to tetracycline, trimethoprim-sulfamethoxazole, and nalidixic acid, respectively. Even though most of the isolate showed different resistance patterns, 16 $(80 \%)$ and $2(10 \%)$ tested isolates were susceptible to nalidixic acid and trimethoprim-sulfamethoxazole, respectively. The antimicrobial resistance in Salmonella is one of the main concerns of its infection in humans [32].

As can be seen from Table 4, Salmonella were resistance to ampicillin (100\%), tetracycline (85\%), and trimethoprimsulfamethoxazole $(80 \%)$, which was comparable to the findings in Harar $(100 \%, 100 \%$, and $85.7 \%$, respectively) as reported by [9] and $100 \%$ and $86.8 \%$ resistant to ampicillin and trimethoprim-sulfamethoxazole as reported by [39] in Pakistan. Moreover, a study from Addis Ababa reported that $81.2 \%, 94.5 \%$, and $75.7 \%$ of the isolate was resistant to ampicillin, tetracycline, and trimethoprim-sulfamethoxazole, respectively [38]. In contrast to the present study, all were found to be susceptible to ampicillin, tetracycline, and trimethoprim-sulfamethoxazole in a study at Hawassa by [11].

The present finding was higher than the previous studies reported by [7], which showed that a high frequency of resistance in Salmonella isolates was observed to tetracycline (52.5\%), cotrimoxazole (37.5\%), and ampicillin (60\%). Moreover, $80 \%$ of the isolate had resistance to ampicillin according to [10]. The rate of resistance of Salmonella isolates to tetracycline in present study (85\%) was higher than in Harar (71.4\%) [37] and in Mozambique (15\%) [40].

Resistance to ampicillin and trimethoprim-sulfamethoxazole in the present study was also higher than those 
TABle 3: Associations of Salmonella infection and clinical features in diarrheal patients.

\begin{tabular}{|c|c|c|c|c|c|}
\hline Symptoms & Positive (\%) & Negative (\%) & $X^{2}$ & Df & $P$ values \\
\hline \multicolumn{6}{|l|}{ Fever } \\
\hline Yes & $14(5.5)$ & $238(94.5)$ & \multirow{2}{*}{3.200} & \multirow{2}{*}{1} & \multirow{2}{*}{0.074} \\
\hline No & $6(4.5)$ & $126(95.5)$ & & & \\
\hline \multicolumn{6}{|l|}{ Vomiting } \\
\hline Yes & $16(6.8)$ & $220(93.2)$ & \multirow{2}{*}{7.200} & \multirow{2}{*}{1} & \multirow{2}{*}{$0.007^{* * *}$} \\
\hline No & $4(2.7)$ & $144(97.3)$ & & & \\
\hline \multicolumn{6}{|l|}{ Abdominal pain } \\
\hline Yes & $18(6.9)$ & $241(93.1)$ & \multirow{2}{*}{12.800} & \multirow{2}{*}{1} & \multirow{2}{*}{$0.000^{* * *}$} \\
\hline No & $2(1.6)$ & $123(98.4)$ & & & \\
\hline \multicolumn{6}{|l|}{ Consistency of stool } \\
\hline Watery & $12(6.9)$ & $162(93.1)$ & \multirow{4}{*}{13.600} & \multirow{4}{*}{3} & \multirow{4}{*}{$0.004^{* * *}$} \\
\hline Bloody & $2(4.8)$ & $40(95.2)$ & & & \\
\hline Mucoid & $4(4)$ & $96(96)$ & & & \\
\hline Mixed (blood and mucus) & $2(2.9)$ & $66(97.1)$ & & & \\
\hline \multicolumn{6}{|l|}{ Duration of diarrhea (days) } \\
\hline $1-5$ & $12(3.9)$ & $294(96.1)$ & \multirow{4}{*}{7.600} & \multirow{4}{*}{2} & \multirow{4}{*}{$0.022^{* * *}$} \\
\hline $6-10$ & $6(9.7)$ & $56(90.3)$ & & & \\
\hline $11-15$ & $2(16.7)$ & $10(83.3)$ & & & \\
\hline$\geq 16$ days & 0.0 & $4(100)$ & & & \\
\hline
\end{tabular}

*** Significant at $P<0.05 ; X^{2}$, chi-square; Df, degree of freedom.

Table 4: Drug resistance/susceptibility pattern of Salmonella species isolated in thepresent study $(n=20)$.

\begin{tabular}{lccccc}
\hline Susceptibility & AMP no. (\%) & TRA no. (\%) & SXT no. (\%) & NA no. (\%) & CI no. (\%) \\
\hline$R$ & $20(100 \%)$ & $17(85 \%)$ & $16(80 \%)$ & $3(15 \%)$ & 0 \\
$I$ & 0 & $3(15 \%)$ & $2(10 \%)$ & $1(5 \%)$ & 0 \\
$S$ & 0 & 0 & $2(10 \%)$ & $16(80 \%)$ & $20(100 \%)$ \\
\hline
\end{tabular}

AM, ampicillin; CI, ciprofloxacin; SXT, trimethoprim-sulphamethoxazole; NA, nalidixic acid; TRA, tetracycline; $S$, sensitive; $I$, intermediate; $R$, resistance.

TABle 5: Antimicrobial resistance patterns of Salmonella in the present study and similar other research studies in different parts of Ethiopia.

\begin{tabular}{|c|c|c|c|c|c|c|c|}
\hline \multicolumn{8}{|c|}{ Study area with respective study periods } \\
\hline Antibiotic & Jimma $^{1}$ & Addis $\mathrm{Ababa}^{2}$ & Harar $^{3}$ & Jimma and Addis Ababa ${ }^{4}$ & Harar $^{5}$ & Butajira $^{6}$ & Dessie $^{*}$ \\
\hline $\mathrm{AM}$ & 59.3 & 81.3 & 100 & 82.3 & 100 & 60 & 100 \\
\hline CI & $\mathrm{Nd}$ & $\mathrm{Nd}$ & $\mathrm{Nd}$ & 0.9 & 28 & 2.5 & 0.0 \\
\hline SXT & 40.7 & 75.7 & $\mathrm{Nd}$ & 80.5 & $\mathrm{Nd}$ & 37.5 & 80 \\
\hline NA & 8.5 & 37.8 & $\mathrm{Nd}$ & 8.0 & 3 & 5 & 15 \\
\hline TRA & 59.3 & 94.5 & 71.4 & 39.8 & 100 & 52.5 & 85 \\
\hline
\end{tabular}

1, [3]; 2, [38]; 3, [37]; 4, [13]; 5, [9]; 6, [7]; *the present study; Nd, no data; AM, ampicillin; CI, ciprofloxacin; SXT, trimethoprim-sulfamethoxazole; NA, nalidixic acid; TRA, tetracycline.

reported from Brazil, where only $88.8 \%$ and $56.8 \%$ were found to be resistant, respectively [39]. But $86.4 \%$ resistant to tetracycline is comparable with the present study.

The increased resistance towards ampicillin (100\%), tetracycline (85\%), and trimethoprim-sulfamethoxazole $(80 \%)$ in this study might be due to misuse of those antibiotics in the study area. It was revealed that antibiotic resistance of Salmonella isolates from diarrheic children in Southeastern Africa was conferred by TEM-like $\beta$-lactamases for ampicillin, floR genes, and CAT activity for chloramphenicol, tetA genes for tetracycline, and $d f r A 1$ genes for trimethoprim-sulfamethoxazole/cotrimoxazole [40].

In the present study, $100 \%$ and $80 \%$ of Salmonella isolates were shown to be susceptible to ciprofloxacin and nalidixic acid, respectively, and they can be used to treat salmonellosis in the study area. The present finding was in line with a study conducted in Harar by [9].

Table 5 shows findings from different regions and periods, such as central Ethiopia (Addis Ababa), Southern Ethiopia (Butajira), southwest Ethiopia (Jimma), Eastern Ethiopia (Harar), and Dessie (the present study). The data revealed that the highest percentages of ampicillin and tetracycline resistant Salmonella isolates were reported from Harar (100\% each), Addis Ababa (81.3\% and $94.5 \%)$ in 2008, and Dessie (100\% and $85 \%)$. Similarly, an increased resistance of trimethoprim-sulphamethoxazole was documented in Jimma and Addis Ababa [13], Dessie (the present study), and Addis Ababa [38], where $80.5 \%, 80 \%$, and $75.7 \%$ have been shown to be resistant to this antibiotic, respectively. 
Resistance against trimethoprim-sulfamethoxazole and tetracycline was reported from Jimma [3] and Jimma and Addis Ababa [13] where $40.7 \%$ and $39.8 \%$, respectively, were found to be resistant to those antibiotics.

\section{Conclusion}

The result of the present study showed that the prevalence of salmonellosis among diarrheal patients who visited Dessie Referral Hospital, Dessie, Northeast Ethiopia, from November to January 2017 was $5.21 \%$. This prevalence rate of Salmonella species is associated with drinking unprotected water, consumption of raw vegetable and fruits, consumption of raw meat, and absence of latrine in the households. Relatively, its prevalence was lower for those study subjects that had safe and adequate drinking water and proper excreta disposal systems.

Prevention of salmonellosis depends primarily on measures that prevent the spread of the organism in the community and prevent the spread from person to person through washing hand with soap after toilet, by drinking protected water, and proper handling of foods. These measures are not only used for salmonellosis but are also used for other diarrheal diseases. In nutshell, creating awareness about transmission and risk factors of Salmonella is necessary.

In this study, $100 \%$ of the isolate was susceptible to ciprofloxacin, so it may be used for the treatment of salmonellosis. Besides, tetracycline, ampicillin, and cotrimoxazole should not be used for the treatment of Salmonella infection unless culture and sensitivity tests are performed prior to treatment.

\section{Recommendations}

The following recommendations are made based on the finding:

The present study did not differentiate Salmonella isolate into the species level. Therefore, further studies that aim to differentiate serogroups and serotypes of Salmonella are needed.

The present study was conducted in a short period of time from November to January 2017. Additional study is needed to assess the seasonal variation in prevalence and antimicrobial resistance patterns of Salmonella species.

Raw eggs, raw vegetables and fruit, and milk should not be consumed in order to limit the spread of salmonellosis

Pretreatment of water obtained from unprotected sources prior to consumption, the need to improve the hygienic conditions of street vended foods, and reduce and remove too many contact (living with animals) in order to limit the spread of salmonellosis

\section{Data Availability}

The dataset used to support the findings of this study is available from the author upon request.

\section{Conflicts of Interest}

The author declares that there are no conflicts of interest.

\section{Acknowledgments}

The author acknowledges the support provided by Minister of Education of Ethiopia to accomplish this study. The study participants are duly acknowledged for voluntarily supplying the stool sample and responding to the questionnaire. The author expressed his gratitude to data collectors and supervisors for their cooperation.

\section{References}

[1] World Health Organization, Diarrhea, World Health Organization, Geneva, Switzerland, 2017, http://www.who.int/ topics/diarrhoea/en.

[2] C. Langendorf, S. Le Hello, A. Moumouni et al., "Enteric bacterial pathogens in children with diarrhea in Niger: diversity and antimicrobial resistance," PLoS One, vol. 10, no. 3, Article ID e0120275, 2015.

[3] A. Mache, "Salmonella serogroups and their antibiotic resistance patterns isolated from diarrhoeal stools of pediatric out-patients in Jimma hospital and Jimma health center, South West Ethiopia," Ethiopian Journal of Health Sciences, vol. 12, no. 1, 2002.

[4] The Centers for Disease Control and Prevention (CDC), Global Health, Center for Disease Control and Prevention $(C D C)$, Centers for Disease Control and Prevention (CDC), Atlanta, GA, USA, 2016, https://www.cdc.gov/globalhealth/ countries/ethiopia/.

[5] F. I. Akinnibosun and F. C. Nwafor, "Prevalence of diarrhea and antibiotic susceptibilitytest in children below 5 years at university of Benin teaching hospital, Nigeria," International Research Journal Publication and Environmental Health, vol. 2, no. 4, pp. 49-55, 2015.

[6] S. D. Puthucheary, K. P. Ng, A. Hafeez, N. S. Raja, and H. H. Hassan, "Salmonellosis inpersons infected with human immunodeficiency virus: a report of seven cases from Malaysia," The Southeast Asian Journal of Tropical Medicine and Public Health, vol. 35, no. 2, pp. 361-365, 2004.

[7] G. Mengistu, G. Mulugeta, T. Lema, and A. Aseffa, "Prevalence and antimicrobial susceptibility patterns of Salmonella serovars and Shigella species," Journal of Microbial and Biochemical Technology, vol. 2, p. 006, 2014.

[8] G. Beyene and H. Tassew, "Prevalence of intestinal parasite, Shigella and Salmonella species among diarrheal children in Jimma health center, Jimma southwest Ethiopia," Annals of Clinical Microbiology and Antimicrobials, vol. 13, pp. 1-7, 2014.

[9] D. Abebe, A. Kebede, and S. Menkir, "Prevalence of antibiotic resistant Salmonella isolates Entamoeba histolytica and Giardia lamblia in Harar, Eastern Ethiopia," African Journal of Microbiology Research, vol. 8, no. 20, pp. 2044-2053, 2014.

[10] Y. Mamuye, G. Metaferia, A. Birhanu, K. Desta, and S. Fantaw, "Isolation and antibiotic susceptibility patterns of Shigella and Salmonella among under 5 children with acute diarrhoea: a cross-sectional study at selected public health facilities in Addis Ababa, Ethiopia," Clinical Microbiology: Open Access, vol. 4, no. 1, 2015.

[11] M. Getamesay, B. Getenet, and Z. Ahmed, "Prevalence of Shigella, Salmonella and Campylobacter species and their 
susceptibility patters among under five children with diarrhea in Hawassa town, south Ethiopia," Ethiopian Journal of Health Science, vol. 24, no. 2, pp. 101-108, 2014.

[12] R. M. Humphries and A. J. Linscott, "Laboratory diagnosis of bacterial gastroenteritis," Clinical Microbiology Revision, vol. 28, no. 1, pp. 3-31, 2015.

[13] G. Beyene, S. Nair, D. Asrat et al., "Multidrug resistant Salmonella concord is a major cause of salmonellosis in children," The Journal of Infection in Developing Countries, vol. 5, no. 1, pp. 23-33, 2011.

[14] M. Bland, An Introduction to Medical Statistics, Oxford University Press, London, UK, 1st edition, 1998.

[15] M. Cheeseborough, District Laboratory Practise in Tropical Countries, Cambridge University Press, Cambridge, NY, USA, Part Two, 4th edition, 2006.

[16] Clinical and Laboratory Standards Institute Editor, Performance Standards for Antimicrobial Susceptibility Testing, Clinical and Laboratory Standards Institute, Wayne, NJ, USA, 27th edition, 2017.

[17] S. Xia, S. R. Hendriksen, Z. Xie et al., "Molecular characterization and antimicrobial susceptibility of Salmonella in humans in henan province, China," Journal of Clinical Microbiology, vol. 13, pp. 421-433, 2008.

[18] I. A. Mikhail, E. Fox, R. L. Haberberger, M. H. Ahmed, and E. A. Abbatte, "Epidemiology of bacterial pathogens associatedwith infectious diarrhoea in Djibouti," Journal of Clinical Microbiology, vol. 28, no. 5, pp. 956-961, 1990.

[19] T. I. Ogunsanya, V. O. Rotimi, and A. Adenuga, "A study of the aetiological agents of childhood diarrhoea in Lagos, Nigeria," Journal of Medical Microbiology, vol. 40, no. 1, pp. 10-14, 1994.

[20] E. M. Urio, E. K. Collison, B. A. Gashe, T. K. Sebunya, and S. Mpuchane, "Shigella and Salmonella strains isolated from children under 5 years in Gaborone, Botswana, and their antibiotic susceptibility patterns," Tropical Medicine and International Health, vol. 6, no. 1, pp. 55-59, 2001.

[21] T. Andualem, M. Tiruneh, F. Moges, D. Muluye, and G. Ayalew, "Prevalence and antimicrobial susceptibility patterns of Shigella and Salmonella species among patients with diarrhea attending gondar town health institutions, northwest Ethiopia," Science Journal of Public Health, vol. 2, no. 5, pp. 469-475, 2014.

[22] B. Mussaret, P. Zaidi, C. Fedorka-Cray, K. H. Sunnah, H. J. Abbott, and L. Magda, "Nontyphoidal Salmonella from human clinical cases, asymptomatic children, and raw retail meats in Yucatan, Mexico," Clinical Infectious Diseases, vol. 54, no. 12, 2012.

[23] K. O. Akinyemi, B. S. Bamiro, and A. O. Coker, "Salmonellosis in Lagos, Nigeria: incidence of Plasmodium falciparum associated co infection, patterns of antimicrobial resistance, and emergence of reduced susceptibility to fluoroquinolones," Journal of Health Population and Nutrition, vol. 25, no. 3, pp. 351-358, 2007.

[24] V. Curtis and S. Cairncross, "Effect of washing hands with soap on diarrhoea risk in the community: a systematic review," The Lancet Infectious Diseases, vol. 3, no. 5, pp. 275-281, 2003.

[25] D. V. Guane, J. F. Walbog, A. R. Mogran, and L. K. Javson, "Antibiotic resistance in Salmonella typhi and its risk factors," Journal of Clinical Microbiology, vol. 28, pp. 231-237, 2000.

[26] A. Moreira and A. Lima, "Tropical diarrhoea: new developments in traveler's diarrhoea," Current Opinion in Infectious Disease, vol. 14, no. 5, pp. 547-552, 2001.
[27] M. Elbediwi, H. Pan, S. Biswas, Y. Li, and M. Yue, "Emerging colistin resistance in Salmonella enterica serovar newport isolates from human infections," Emerging Microbes \& Infections, vol. 9, no. 1, pp. 535-538, 2020.

[28] L. Lind, J. Reeser, K. Stayman et al., "Salmonella typhimurium infection associated with raw milk and cheese consumptionPennsylvania," MMWR. Morbidity and Mortality Weekly Report, vol. 56, no. 44, pp. 1161-1164, 2007.

[29] B. M. Jayarao, S. C. Donaldson, B. A. Straley, A. A. Sawant, N. V. Hegde, and J. L. Brown, "A survey of food borne pathogens in bulk tank milk and raw milk consumption among farm families in Pennsylvania," Journal of Dairy Science, vol. 89, no. 7, pp. 2451-2458, 2006.

[30] B. Bouchrif, B. Paglietti, M. Murgia et al., "Prevalence and antibiotic-resistance of Salmonella isolated from food in Morocco," Journal of Infection in Developing Countries, vol. 28, no. 3, pp. 35-40, 2009.

[31] P. K. Feglo, E. H. Frimpong, and M. Essel-Ahun, "Salmonella carrier status of food vendors in Kumasi, Ghana," East African Medical Journal, vol. 81, no. 7, pp. 358-361, 2004.

[32] D. Gu, Z. Wang, Y. Tian et al., "Prevalence of Salmonella isolates and their distribution based on whole-genome sequence in a chicken slaughterhouse in Jiangsu, China," Frontiers in Veterinary Science, vol. 7, p. 29, 2020.

[33] E. Elnekave, S. L. Hong, S. Lim, T. J. Johnson, P. Andres, and J. Alvarez, "Comparing serotyping with whole-genome sequencing for subtyping of non-typhoidal Salmonella enterica: a large-scale analysis of 37 serotypes with a public health impact in the USA," Microbial Genomics, vol. 6, no. 9, 2020.

[34] S. L. Foley and A. M. Lynne, "Food animal associated Salmonella challenges: pathogenicity and antimicrobial resistance," Journal of Animal Science, vol. 86, pp. E173-E187, 2008.

[35] J. A. Crump, K. M. Thomas, J. Benschop et al., "Investigating the meat pathway as a source of human nontyphoidal Salmonella bloodstream infections and diarrhea in East Africa," Clinical Infectious Diseases: An Official Publication of the Infectious Diseases Society of America, pp. 1-9, 2020.

[36] T. Nagy, A. Szmolka, T. Wilk et al., "Comparative genome analysis of Hungarian and global strains of Salmonella Infantis," Frontiers in Microbiology, vol. 11, p. 539, 2020.

[37] A. A. Reda, B. Seyoum, J. Yimam, G. Andualem, S. Fiseha, and J. M. Vandeweerd, "Antibiotic susceptibility patterns of Salmonella and Shigella isolates in Harar, Eastern Ethiopia," Journal of Infectious Disease and Immunity, vol. 3, no. 8, pp. 134-139, 2011.

[38] D. Asrat, "Shigella and Salmonella serogroups and their antibiotic susceptibility patterns in Ethiopia," Eastern Mediterranean Health Journal, vol. 14, no. 4, pp. 760-767, 2008.

[39] A. M. Ali, A. H. Qureshi, S. Rafi et al., "Frequency of Salmonella in diarrhoea/dysentery in children in Rawalpindi and Islamabad," Journal of Pakistan Medical Association, vol. 53, no. 11, pp. 517-520, 2003.

[40] I. Mandomando, D. Jaintilal, M. J. Pons et al., "Antimicrobial susceptibility and mechanisms of resistance in Shigella and Salmonella isolates from children under five years of age with diarrhea in rural Mozambique," Antimicrobial Agents Chemotherapy, vol. 53, no. 6, pp. 2450-2454, 2009. 